\title{
Pacific
}

Journal of

Mathematics

\section{ISOPERIMETRIC INEQUALITIES FOR AUTOMORPHISM GROUPS OF FREE GROUPS}

Allen E. Hatcher And Karen Vogtmann 


\title{
ISOPERIMETRIC INEQUALITIES FOR AUTOMORPHISM GROUPS OF FREE GROUPS
}

\author{
Allen Hatcher and Karen VogtmanN
}

\begin{abstract}
In this paper we show that the groups of automorphisms and outer automorphisms of a finitely generated free group have isoperimetric functions which are bounded above by exponential functions. This exponential bound is best possible if the free group has rank three, but the best bound remains unknown in higher rank. Our techniques show more generally that $n$-dimensional isoperimetric functions for these groups are at most exponential for all $n$. A variation of the technique gives an asynchronous bounded combing of the mapping class group of a bounded surface.
\end{abstract}

\section{Introduction.}

The geometric notion of an isoperimetric inequality can be imitated combinatorially in a simply-connected cell complex $X$ as follows. For any loop in the one-skeleton of $X$, its length is the number of times it passes over onecells, and its area is the minimum number of times it passes over two-cells during any null-homotopy. The isoperimetric function $\delta(n)$ measures the maximum area of loops of length $n$ in the one-skeleton. By an isoperimetric inequality we mean an upper bound for $\delta(n)$ in terms of some other function $f(n)$.

This idea can be transferred to finitely presented groups $G$ by taking $X$ to be the universal cover of the standard two-complex associated to a finite presentation of $G$. Different finite presentations of $G$ give rise to quasiisometric complexes, and there is an equivalence relation on functions so that quasi-isometric complexes have equivalent isoperimetric functions $\delta(n)$; see [A]. This equivalence relation identifies all polynomials of a fixed degree, all exponential functions $a^{n}$, all double exponentials $a^{b^{n}}$, etc. Gromov's hyperbolic groups are characterized by the fact that their isoperimetric functions are equivalent to linear functions $[\mathbf{G r}]$. Automatic groups and semi-hyperbolic groups satisfy quadratic isoperimetric inequalities (see [ECHLPT] and [AB]). A group has a solvable word problem if and only if its isoperimetric function is recursive [ECHLPT].

The main result in this paper is the following: 
Theorem 1.1. The groups $\operatorname{Aut}\left(F_{n}\right)$ and $\operatorname{Out}\left(F_{n}\right)$ of automorphisms and outer automorphisms of a finitely generated free group $F_{n}$ satisfy exponential isoperimetric inequalities, i.e. their isoperimetric functions are at most exponential.

It is interesting to compare our result on the isoperimetric functions for $\operatorname{Out}\left(F_{n}\right)$ and $\operatorname{Aut}\left(F_{n}\right)$ with the situation for $G L(n, \mathbf{Z})$. The group $G L(n, \mathbf{Z})$ is related to $\operatorname{Aut}\left(F_{n}\right)$ and $\operatorname{Out}\left(F_{n}\right)$ by the natural map which sends an automorphism of a free group to the induced automorphism on the free abelian group. For $n=2, \operatorname{Out}\left(F_{2}\right)$ is isomorphic to $G L(2, \mathbf{Z})$ and satisfies a linear isoperimetric inequality; this follows from the well-known cocompact action of $G L(2, \mathbf{Z})$ on a tree [S]. It is known that $\operatorname{Aut}\left(F_{2}\right)$ is automatic but not hyperbolic, so has a quadratic isoperimetric function (see [Ge]). For $n=3$, [ECHLPT] show that the isoperimetric function for $G L(3, \mathbf{Z})$ is at least exponential; this implies the same fact for $\operatorname{Aut}\left(F_{3}\right)$ and $\operatorname{Out}\left(F_{3}\right)$, since there is a loop in $G L(3, \mathbf{Z})$ which is exponentially hard to fill and which lifts to a loop in $\operatorname{Aut}\left(F_{3}\right)[\mathbf{B V}]$. For $n \geq 4$, the group $G L(n, \mathbf{Z})$ satisfies a quadratic isoperimetric inequality, according to Thurston (see $[\mathbf{G r 2}]$ ), while the best upper bound for $\operatorname{Aut}\left(F_{n}\right)$ and $\operatorname{Out}\left(F_{n}\right)$ is unknown.

In [ECHLPT] the notion of higher-dimensional isoperimetric function is introduced. Our techniques show that the groups $\operatorname{Aut}\left(F_{n}\right)$ and $\operatorname{Out}\left(F_{n}\right)$ also satisfy exponential higher-dimensional isoperimetric inequalities. The $(n-1)$-dimensional isoperimetric function for $G L(n, \mathbf{Z})$ is at least exponential [ECHLPT], but for $n \geq 4$ the analogous result for $\operatorname{Aut}\left(F_{n}\right)$ and $\operatorname{Out}\left(F_{n}\right)$ remains unknown.

Our methods extend easily to show that mapping class groups of punctured surfaces, which are subgroups of $\operatorname{Out}\left(F_{n}\right)$, also satisfy exponential isoperimetric inequalities in all dimensions. Recently L. Mosher [M1], [M2] has shown that mapping class groups of surfaces (with or without punctures) are actually automatic, so in fact satisfy quadratic isoperimetric inequalities.

In $[\mathbf{G e 2}]$ Gersten showed that $\operatorname{Aut}\left(F_{n}\right)$ and $\operatorname{Out}\left(F_{n}\right)$ satisfy a double exponential isoperimetric inequality. Since then, he has independently obtained exponential isoperimetric inequalities for these groups [private communication].

Our approach to these problems is to consider 2-spheres in an appropriate 3 -manifold $M$. In the case of $\operatorname{Out}\left(F_{n}\right), M$ is the connected sum of $n$ copies of $S^{1} \times S^{2}$, and in the case of $\operatorname{Aut}\left(F_{n}\right), M$ is the same connected sum "punctured" by removing the interior of a 3-ball. Associated to $M$ is a simplicial complex $X=X(M)$ whose vertices are isotopy classes of certain systems $S$ of disjointly embedded 2-spheres in $M$. The complex $X$ is a subcomplex of the barycentric subdivision of the sphere complex $\mathcal{S}(M)$ of 
$[\mathbf{H}]$. In the case of $\operatorname{Out}\left(F_{n}\right), X$ can be identified with the complex of minimal free actions of $F_{n}$ on simplicial trees, as defined in [CV] (also known as the spine of Outer space).

The mapping class group $\pi_{0}\left(\operatorname{Diff}_{+}(M)\right)$ of isotopy classes of orientationpreserving diffeomorphisms of $M$ acts on $X$. The normal subgroup generated by "Dehn twists" along 2-spheres acts trivially, so there is an induced action of the quotient group $\Gamma$ on $X$. By a theorem of Laudenbach $[\mathbf{L}]$, this quotient group is $\operatorname{Aut}\left(F_{n}\right)$ or $\operatorname{Out}\left(F_{n}\right)$ in the punctured and unpunctured cases respectively. The complex $X$ is contractible and locally finite, the action of $\Gamma$ on $X$ has finite simplex-stabilizers, and $X / \Gamma$ is a finite complex. Thus $X$ is quasi-isometric to $\Gamma$, and we can use $X$ to compute an isoperimetric inequality for $\Gamma$.

In $[\mathbf{H}]$ a contraction of the sphere complex $\mathcal{S}(M)$ was constructed by joining each sphere system $S$ to a fixed system $\Sigma$ by a canonical path in $\mathcal{S}(M)$. In case $S$ and $\Sigma$ are vertices of $X$, a combinatorial version of this construction gives a canonical edgepath in $X$ from $S$ to $\Sigma$. This path should be viewed as oriented since the canonical path from $S$ to $\Sigma$ is usually different from the path from $\Sigma$ to $S$. By fixing $\Sigma$ and letting $S$ vary we then obtain a combing of $X$, i.e. a choice of an edgepath from each vertex $S$ to the basepoint vertex $\Sigma$. Similarly, by fixing $S$ and letting $\Sigma$ vary we obtain a second combing of $X$. We call these two combings the inward and outward combings, respectively.

Only the inward combing was studied in $[\mathbf{H}]$, but it turns out that the outward combing has some technical advantages over the inward combing. The outward combing leads to an exponential isoperimetric inequality by letting $\Sigma_{1}, \Sigma_{2}, \ldots, \Sigma_{n}$ be the successive vertices in a loop in $X$ at the basepoint $S$, and then analyzing how to fill in a homotopy between the canonical paths to adjacent vertices $\Sigma_{i}, \Sigma_{i+1}$ along this loop. The homotopies between successive canonical paths then combine to give a contraction of the given loop, and we obtain the exponential isoperimetric inequality by counting simplices in this contraction. Higher-dimensional isoperimetric inequalities are obtained in a similar way.

As a bonus, the outward combing gives a proof of the contractibility of $X$ which seems simpler than the one in $[\mathbf{H}]$, which was based on inward combing. With this simpler proof, the dependence of the present paper on $[\mathbf{H}]$ is reduced to just the existence and uniqueness of "normal forms" for 2-spheres in $M$.

These techniques also apply to mapping class groups of punctured surfaces, using the outward combing for certain sphere systems which correspond to arc systems on the surfaces. A variation of the inward combing, which involves ordering and orienting the base arc system, gives a bounded 
asynchronous combing, i.e. the combing lines starting at adjacent vertices remain a bounded distance apart, when suitably parametrized. Mosher's proof of the automaticity of mapping class groups can be viewed as refining and elaborating on this inward combing. In the case of punctured surfaces he shows that the failure of the combing to be synchronous can be analyzed and then finessed away by cleverly rechoosing generators.

\section{Sphere complexes and normal form.}

As in the introduction, let $M$ be the connected sum of $n$ copies of $S^{1} \times S^{2}$, with perhaps a puncture.

Definition. A sphere set in $M$ is a finite set of disjointly embedded 2spheres in $M$. A sphere set $S$ is simple if every connected component of $M-S$ is simply-connected. A sphere system is a sphere set $S$ such that no two spheres of $S$ are isotopic and no sphere in $S$ is trivial, i.e. bounds a ball or is isotopic to the boundary sphere.

We define complexes $\widehat{X}$ and $X$ as the geometric realizations of the partially ordered sets of isotopy classes of simple sphere sets in $M$ and simple sphere systems in $M$, respectively. We have $X \subset \widehat{X}$, and there is a natural projection from $\widehat{X}$ onto $X$ given by deleting from a sphere set all but one sphere in each isotopy class, as well as all trivial spheres.

Sphere sets $S_{0} \ldots, S_{p}$ are said to be compatible if their union is a sphere set, i.e. if they are disjoint except for common spheres. In this case there is a canonical simplicial map of the barycentric subdivision of a $p$-simplex $\Delta^{p}$ into $\widehat{X}$, taking the vertices of $\Delta^{p}$ to the $S_{i}$ 's and the barycenter corresponding to a subset of the vertices to the sphere set which is the union of the corresponding $S_{i}$ 's. For example, two compatible sphere sets $S$ and $S^{\prime}$ give rise to the path $S \subset\left(S \cup S^{\prime}\right) \supset S^{\prime}$ of length two in $\widehat{X}$.

The mapping class group $\pi_{0}\left(\operatorname{Diff}_{+}(M)\right)$ acts on $\widehat{X}$ preserving $X$. A "Dehn twist" along an $S^{2} \subset M$ is a diffeomorphism supported in a tubular neighborhood $S^{2} \times[0,1]$, taking the slice $S^{2} \times\{t\}$ to itself by a rotation through angle $2 \pi t$ with respect to some chosen axis of $S^{2}$. The effect of such a twist on a sphere set is to produce a new sphere set homotopic to the old one. By a theorem of Laudenbach $[\mathbf{L}]$, homotopic sphere sets are isotopic, so Dehn twists act trivially on $\widehat{X}$ and there is an induced action of the quotient group $\Gamma$ of $\pi_{0}\left(\operatorname{Diff}_{+}(M)\right)$ by the subgroup generated by Dehn twists, which is a normal subgroup. Laudenbach also proved that $\Gamma$ is isomorphic to $\operatorname{Aut}\left(F_{n}\right)$. or $\operatorname{Out}\left(F_{n}\right)$ in the punctured and unpunctured cases, respectively, via the natural map associating to a diffeomorphism of $M$ its induced automorphism of $\pi_{1}(M) \cong F_{n}$.

In $[\mathbf{H}]$, a simplicial complex $\mathcal{S}(M)$ was defined by taking a simplex of 
dimension $k$ for each isotopy class of sphere systems with $k+1$ spheres, with face relations given by omitting spheres from the system. The complex $X(M)$ is the full subcomplex of the barycentric subdivision of $\mathcal{S}(M)$ spanned by sphere systems which are simple. A key ingredient for the study of the sphere complex $\mathcal{S}(M)$ was the notion of "normal form" for a sphere system $S$ with respect to a maximal sphere system $\Sigma$. We need to extend this idea to the case of simple systems $\Sigma$. (Maximal systems are necessarily simple.)

Definition. Let $\Sigma$ be a simple sphere system. A sphere system $S$ is in normal form with respect to $\Sigma$ if for each sphere $s$ of $S$, one of three possibilities holds: (a) $s$ coincides with a sphere of $\Sigma$; (b) $s$ is disjoint from $\Sigma$ and not isotopic to any sphere of $\Sigma$; (c) $s$ has non-empty transverse intersection with $\Sigma$ and, for each component $P$ of $M-\Sigma$,

(i) each component of $s \cap P$ has at most one boundary circle on each boundary sphere of $P$ and

(ii) no component of $s \cap P$ is a trivial disk, isotopic in $P$ to a disk in the boundary of $P$.

Note that (b) can occur only if $\Sigma$ is not a maximal system.

Proposition 2.1. Let $\Sigma$ be a simple sphere system. Every sphere system $S$ is isotopic to a sphere system which is in normal form with respect to $\Sigma$.

Proof. This was shown in $[\mathbf{H}]$ for maximal systems $\Sigma$. The general case follows since a given $\Sigma$ can always be enlarged to a maximal system $\Sigma^{\prime}$, and if $S$ is in normal form with respect to $\Sigma^{\prime}$ it is in normal form with respect to $\Sigma$.

Two sphere systems $S$ and $S^{\prime}$ in normal form with respect to $\Sigma$ are equivalent if there is a homotopy $S_{t}$ from $S_{0}=S$ to $S_{1}=S^{\prime}$ which is fixed on the common spheres of $S$ and $\Sigma$ and which on the others is transverse to $\Sigma$ at all times, with the circles of $S_{t} \cap \Sigma$ varying only by isotopy in $\Sigma$.

Proposition 2.2. Two isotopic sphere systems $S$ and $S^{\prime \prime}$ in normal form with respect to a simple system $\Sigma$ are equivalent.

Proof. It suffices to show that $S$ is equivalent to a system in normal form with respect to a maximal $\Sigma^{\prime} \supset \Sigma$, and likewise for $S^{\prime}$, since the case of maximal $\Sigma$ was proved in $[\mathbf{H}]$. The procedure in $[\mathbf{H}]$ for putting systems into normal form has the property that if $S$ is already in normal form with respect to $\Sigma$, then putting it into normal form with respect to $\Sigma^{\prime} \supset \Sigma$ does not change its equivalence class with respect to $\Sigma$. Namely, one looks in each component $P$ of $M-\Sigma^{\prime}$ to see whether conditions (i) and (ii) are satisfied. If either condition fails, one is able to decrease the number of circles of 
$S \cap \Sigma^{\prime}$ by certain moves which are of three sorts: ambient isotopy of the pair $\left(M, \Sigma^{\prime}\right)$; homotopy of $S$ taking place in the complement of $\Sigma^{\prime}$; and isotopy of $S$ supported in a neighborhood of the boundary sphere of $P$ for which (i) or (ii) fails. It is only the third move which decreases the number of circles of $S \cap \Sigma^{\prime}$. The first two moves do not affect the equivalence class of $S$ with respect to either $\Sigma$ or $\Sigma^{\prime}$. Moves of the third type can only involve spheres of $\Sigma^{\prime}-\Sigma$ if $S$ is already in normal form with respect to $\Sigma$, so these moves also do not change the equivalence class of $S$ with respect to $\Sigma$.

\section{Innermost surgery and combing paths.}

Let $\Sigma$ be a simple sphere system, and $S$ a sphere set intersecting $\Sigma$ transversely except for common spheres. We describe a procedure for producing a new sphere set which intersects $\Sigma$ in fewer circles. Let $C$ be a circle component of $S \cap \Sigma$ which is innermost in $\Sigma$, i.e. $C$ bounds a disk $D \subset \Sigma$ with $D \cap S=C$. If $s$ is the sphere of $S$ containing $C$, take a parallel disjoint copy of $s$ which intersects $D$, then perform surgery on this copy to obtain two new spheres $s^{\prime}$ and $s^{\prime \prime}$ which do not intersect $D$ (see Figure 1). Replacing $s$ by $s^{\prime} \cup s^{\prime \prime}$, we obtain a new sphere set $S^{\prime}$ which is compatible with $S$.
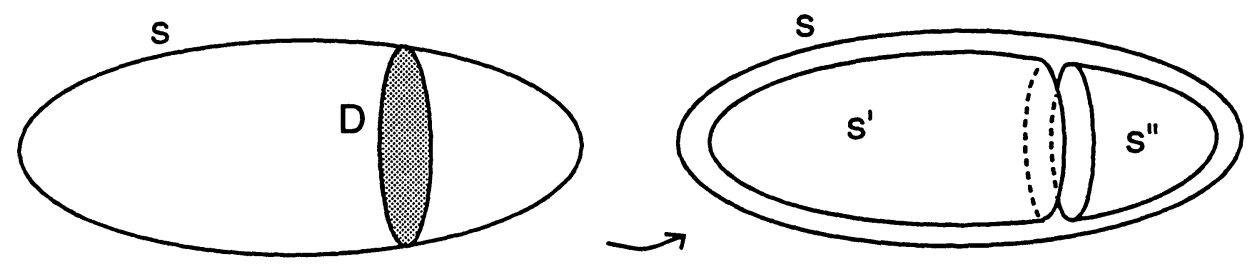

Figure 1.

Lemma 3.1. If $S^{\prime}$ is obtained from a simple sphere set $S$ by surgery along a disk $D$, then $S^{\prime}$ is simple.

Proof. The effect on the complementary components of $M-S$ of surgery along the disk $D$ is that the component containing $D$ is split along $D$ into two components, and the component of $M-S$ on the other side of $S$ from $D$ is changed by the attachment of a 2 -handle. Neither operation introduces non-trivial fundamental group.

Thus, for simple $S$, the sphere sets obtained by first adding $s^{\prime}$ and $s^{\prime \prime}$ to $S$ and then by deleting $s$ describe a path of length two starting from $S$ in the 
complex $\widehat{X}$. The endpoint $S^{\prime}$ of this path has one fewer circle of intersection with $\Sigma$ than $S$ does.

We would like to make this surgery process canonical by performing surgery using all of the innermost circles of $S \cap \Sigma$ at once. This presents no problem when all the innermost circles in each sphere $s$ of $S$ have their disks $D$ lying on the same side of $s$ : we perform all of the surgeries on a single parallel copy of $s$ which intersects all of the disks. We add the resulting spheres to $S$ and then delete the original spheres $s$ to obtain a new system $S^{\prime}$ which is compatible with $S$. We say $S^{\prime}$ is obtained from $S$ by innermost surgery.

A problem occurs if two of the surgery disks lie on opposite sides of some sphere $s$, so that a parallel copy of $s$ will not intersect all of the disks. One might try to fix this by using two parallel copies of $s$, one on each side. Surgery on both of these will produce a sphere set compatible with $S$; however, the total number of intersection circles may increase instead of decreasing after surgery, since the non-innermost circles of $S \cap \Sigma$ have also been duplicated. (One could also try to do surgery on the original sphere $s$ from both sides, but this produces at least one sphere which is not compatible with s.) Another problem occurs when $S$ intersects a sphere of $\Sigma$ in a single circle, since there is then an ambiguity in the choice of the disk $D$. We say $S$ is $\Sigma$-oriented if neither of these two problems occurs, i.e. all surgery disks lie on the same side of each sphere of $S$, and $S$ intersects no sphere of $\Sigma$ in a single circle.

There is a trick which insures $\Sigma$-orientation: instead of starting at $S$, add a parallel copy of each sphere in $S$ which is not in $\Sigma$ to obtain a sphere set $\widehat{S}$. Then we have the following:

Proposition 3.2. Let $\Sigma$ be a simple sphere system, and $S$ a sphere set transverse to $\Sigma$ except for common spheres. Then $\widehat{S}$ is $\Sigma$-oriented, and any sphere set obtained from $\widehat{S}$ by a sequence of innermost surgeries is $\Sigma$ oriented.

Proof. Let $T(S)$ be the finite graph whose vertices are the components of $\Sigma-S$, and whose edges are the circle components of $\Sigma \cap S$. Then $T(S)$ is a disjoint union of trees, one for each sphere of $\Sigma$ which meets $S$ transversely, and $T(\widehat{S})$ is the barycentric subdivision of $T(S)$. Assign a transverse orientation to each sphere of $\widehat{S}$ pointing away from its twin. This induces orientations on the edges of $T(\widehat{S})$. The circles of $\widehat{S} \cap \Sigma$ which we would like to surger in the first innermost surgery correspond to the extremal edges of $T(\widehat{S})$. These edges are all oriented outward, toward the extremal vertices of $T(\widehat{S})$, and the surgery disks are all on the positive side of $\widehat{S}$. So $\widehat{S}$ is $\Sigma$ oriented and the innermost surgery is defined, yielding a sphere set $S_{1}$ with 
$T\left(S_{1}\right)$ obtained from $T(\widehat{S})$ by pruning off all extremal edges. In $T\left(S_{1}\right)$, all of the extremal edges are oriented inward, away from the extremal vertices, and the surgery disks are all on the negative side of $S_{1}$; in particular, $S_{1}$ is $\Sigma$-oriented and we can do innermost surgery to produce a sphere set $S_{2}$. This pattern continues: passing from $S_{i}$ to $S_{i+1}$, the extremal edges pruned off are all oriented in the same way, outward or inward depending on the parity of $i$. This means that all surgeries on $S_{i}$ are performed using disks $D$ on the same side of $S_{i}$ with respect to the transverse orientation.

If $S$ is a simple sphere system in normal form with respect to $\Sigma$, the sequence of sphere sets $\widehat{S}=S_{0}, S_{1}, S_{2}, \ldots$ obtained by successive innermost surgeries determines a path in $\widehat{X}$. The number of intersection circles of $S_{i}$ with $\Sigma$ decreases at each step, so that the path terminates at a set $S_{k}$ which is compatible with $\Sigma$. Thus $S_{k} \cup \Sigma$ is a sphere set, and $S_{k} \subset\left(S_{k} \cup \Sigma\right) \supset \Sigma$ completes a path in $\widehat{X}$ from $\widehat{S}$ to $\Sigma$, called the canonical path. We define the combing path in $X$ from $S$ to $\Sigma$ to be the projection of the canonical path.

Proposition 3.3. If $S$ and $S^{\prime}$ are equivalent sphere systems, the combing path from $S$ to $\Sigma$ in $X$ is the same as the combing path from $S^{\prime}$ to $\Sigma$.

Proof. An equivalence of $S$ involves only isotopy of $S \cap \Sigma$ and homotopy of $S$ within each component of $M-\Sigma$, so the surgeries on equivalent sets correspond bijectively and produce equivalent sets.

The following proposition compares the lengths of the canonical paths to adjacent vertices of $X$.

Proposition 3.4. Fix a vertex $S$ of $X$ and let $\Sigma \subset \Sigma^{\prime}$ be adjacent sphere systems in $X$. Let $k$ and $k^{\prime}$ be the number of surgery steps in the canonical paths in $\widehat{X}$ from $\widehat{S}$ to $\Sigma$ and $\Sigma^{\prime}$ respectively. Then $k \leq k^{\prime} \leq 2 n k+2 n-3$.

Proof. The number $k^{\prime}$ is equal to one-half of the maximal length of a chain of nested circles of $\widehat{S} \cap \Sigma^{\prime}$ on any sphere in $\Sigma^{\prime}$. Let $C_{1}, \ldots, C_{2 k^{\prime}}$ be such a maximal chain of circles, lying on the sphere $\sigma^{\prime}$ of $\Sigma^{\prime}$. If $\sigma^{\prime}$ is a sphere of $\Sigma$, then $k^{\prime}=k$. If $\sigma^{\prime}$ is not in $\Sigma$ then $\sigma^{\prime}$ lies in one component $P$ of $M-\Sigma$, which is a punctured 3-ball.

We assign to each circle $C_{i}$ the component $Q_{i}$ of $\widehat{S} \cap P$ containing $C_{i}$. If $Q_{i}$ does not intersect the boundary of $P$, then $Q_{i}$ is one of the spheres of $\widehat{S}$; since it intersects $\sigma^{\prime}$, it partitions the spheres of the boundary of $P$ into two sets, each with at least two elements. The length of a nested chain of such spheres is at most $2(p-3)$, where $p$ is the number of boundary components of $P$, since no two spheres of $S$ are isotopic. For each sphere $\sigma \in \partial P$, the sequence $\sigma \cap Q_{i}$ is a chain of nested circles in $\sigma \cap \widehat{S}$, so has length at most 
$2 k$. Therefore the length $2 k^{\prime}$ of the chain $\left\{C_{i}\right\}$ is at most $2 p k+2 p-6$. Since $p \leq 2 n$, we get the desired inequality $k^{\prime} \leq 2 n k+2 n-3$.

Remark. The linear estimate for $k^{\prime}$ in terms of $k$ given in Proposition 3.4 can be improved by more careful analysis, to approximately $k^{\prime} \leq(1.7) k+$ $2 n-3$. This still results in an exponential isoperimetric inequality for the group.

\section{Isoperimetric inequalities.}

In this section we prove our main theorem, that the complex $X$ and the groups $\operatorname{Out}\left(F_{n}\right)$ and $\operatorname{Aut}\left(F_{n}\right)$ satisfy exponential isoperimetric inequalities in all dimensions. The notion of higher-dimensional isoperimetric functions was introduced in [ECHLPT], and generalizes the standard isoperimetric function in dimension two. Given a simplicial map of a $(p-1)$-sphere into a $(p-1)$-connected simplicial complex $K$, we define its area to be the number of $(p-1)$-simplices in the domain, and its volume to be the minimal number of $p$-simplices in the domain of a simplicial null-homotopy. The $p$-dimensional isoperimetric function $\delta_{p}(n)$ measures the maximum volume of $(p-1)$-spheres with area at most $n$.

We will construct null-homotopies by a coning operation. We begin by coning off a simplex. In the case $p=2$, the construction is illustrated in Figure 2.

Let $\Sigma_{1} \subset \cdots \subset \Sigma_{p}$ be a simplex in $X$ and let $S$ be a fixed vertex of $X$. Our objective is to construct a map $f: \Delta^{p} \rightarrow X$ of the standard $p$-simplex $\Delta^{p}=\left[v_{0}, \ldots, v_{p}\right]$ into $X$, such that:

(i) $f$ is simplicial with respect to some subdivision of $\Delta^{p}$

(ii) $f\left(v_{0}\right)=S, f\left(v_{i}\right)=\Sigma_{i}$ for $i>0$

(iii) the edge $\left[v_{0}, v_{i}\right]$ maps to the combing path from $S$ to $\Sigma_{i}$, and

(iv) $f$ is linear on the face $\left[v_{1}, \ldots, v_{p}\right]$.

The construction will be canonical, so that the restriction of $f$ to a face $\left[v_{0}, \ldots, \widehat{v}_{i}, \ldots, v_{p}\right]$ with $i>0$ will be the $f$ constructed for the $(p-1)$ simplex $\Sigma_{1} \subset \cdots \subset \widehat{\Sigma}_{i} \subset \cdots \subset \Sigma_{p}$. Hence the $f$ 's will fit together to give a contraction of $X$ to the vertex $S$.

Let $\Sigma_{i}^{\prime}$ denote the sphere set $\Sigma_{i}-\Sigma_{i-1}$, with $\Sigma_{1}^{\prime}=\Sigma_{1}$. From the sphere set $\widehat{S}$ we construct a sphere set $S_{j_{1}, \ldots, j_{p}}$ by performing the first $j_{i}$ innermost surgeries on $\widehat{S}$ with respect to $\Sigma_{i}^{\prime}$, for $i=1, \ldots, p$. We can regard $j_{i}$ as varying from 0 to $\infty$ by letting $S_{j_{1}, \ldots, j_{p}}$ be independent of $j_{i}$ once all the circles of $\widehat{S} \cap \Sigma_{i}^{\prime}$ have been surgered away. Placing $S_{j_{1}, \ldots, j_{p}}$ at the lattice point $\left(j_{1}, \ldots, j_{p}\right)$ in $\mathbf{R}^{p}$, we would like to make these lattice points the vertices of a triangulation of the first orthant of $\mathbf{R}^{p}$ so that the sphere sets $S_{j_{1}, \ldots, j_{p}}$ at 
the vertices of each simplex are all compatible.

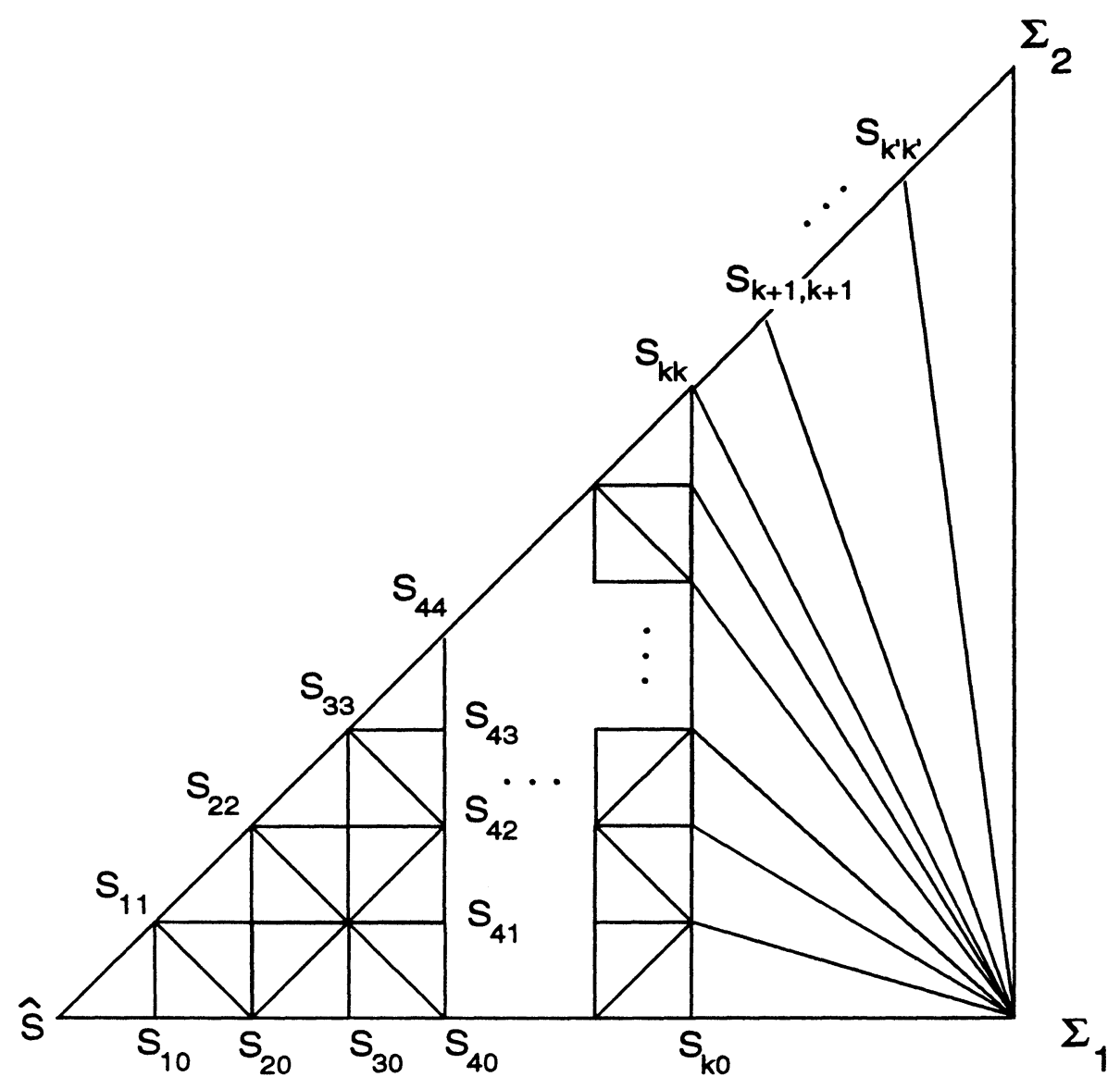

Figure 2.

Consider first the cube $C$ of side length 2 and corners $\left(j_{1}, \ldots, j_{p}\right)$, with each $j_{i}=0$ or 2 . At the center of $C$ is $S_{1, \ldots, 1}$. We can do a sequence of $p$ innermost surgeries of $S_{1, \ldots, 1}$ along the sphere sets $\Sigma_{i}^{\prime}$ in any of the $p$ ! possible orderings of the indices $i=1, \ldots, p$, producing sphere sets $S_{1, \ldots, 1}=$ $S_{0}, S_{1}, \ldots, S_{p}=S_{2, \ldots, 2}$ at lattice points in the cube. All these surgeries use disjoint surgery disks on the negative side of $S_{1, \ldots, 1}$, so the sphere sets $S_{l}$ are all compatible. The corresponding lattice points span a $p$-simplex in the unit subcube of $C$ with main diagonal joining $S_{1, \ldots, 1}$ and $S_{2, \ldots, 2}$, and the $p$ ! simplices of this type form a triangulation of this subcube.

There are $2^{p}-1$ other unit subcubes of $C$ with main diagonals joining $S_{1, \ldots, 1}$ to the other corners of $C$. Each of these subcubes is also triangulated into $p$ ! $p$-simplices with vertices the sequences $S_{1, \ldots, 1}=S_{0}, S_{1}, \ldots, S_{p}$ 
obtained by choosing an ordering of $\Sigma_{1}^{\prime}, \ldots, \Sigma_{p}^{\prime}$ and for each $\Sigma_{i}^{\prime}$ in turn, either doing or undoing an innermost surgery. "Undoing" a surgery along a disk is in fact doing a surgery along an arc. Again all these surgeries are along disjoint disks and arcs on the negative side of $S_{1, \ldots, 1}$, so the sphere sets $S_{0}, \ldots, S_{p}$ are all compatible. Thus we have a triangulation of $C$ into $2^{p} p$ ! $p$-simplices. Abstractly, it is obtained by coning off a triangulation of the boundary of $C$ to the center of $C$, where the triangulation of each $(p-1)$ dimensional cube face of $C$ is triangulated inductively by the same coning procedure.

The same argument provides a triangulation of each cube of side length 2 having corners at lattice points $\left(j_{1}, \ldots, j_{p}\right)$ with each $j_{i}$ even and center at a lattice point $\left(j_{1}, \ldots, j_{p}\right)$ with all $j_{i}$ odd. These triangulations agree on common faces and give a triangulation of the cubical lattice with vertices at the points $\left(j_{1}, \ldots, j_{p}\right)$.

For a fixed integer $k>0$, the simplices spanned by vertices $S_{j_{1}, \ldots, j_{p}}$ with $k \geq j_{1} \geq \cdots \geq j_{p}$ fill up a large $p$-simplex $\Delta$ whose edges emanating from the vertex $\widehat{S}=S_{0, \ldots, 0}$ are initial segments of the canonical paths from $\widehat{S}$ to the systems $\Sigma_{i}$. Specifically, the sphere sets $S_{j_{1}, \ldots, j_{p}}$ where $j_{1}=\ldots=j_{i}$ and $j_{i+1}=\ldots=j_{p}=0$ are the first, third, fifth, etc. vertices in the canonical path from $\widehat{S}$ to $\Sigma_{i}$. Let $k_{i}$ be the number of surgery steps in the canonical path from $\widehat{S}$ to $\Sigma_{i}$, so $k_{1} \leq \cdots \leq k_{p}$ since $\Sigma_{1} \subset \cdots \subset \Sigma_{p}$. Choosing $k=k_{1}$, the sphere sets $S_{j_{1}, \ldots, j_{p}}$ with $j_{1}=k$ form the face of $\Delta$, opposite the vertex $\widehat{S}$, and all of the sphere sets in this face are disjoint from $\Sigma_{1}$.

We embed $\Delta$ linearly in $\Delta^{p}$, taking $S_{0, \ldots, 0}$ to $v_{0}$ and $S_{k, \ldots, k, 0, \ldots, 0}$ with $i k^{\prime} s$ to the midpoint $m_{i}$ of the edge $\left[v_{0}, v_{i}\right]$. The complement of $\Delta$ in $\Delta^{p}$ is a prism $P=P\left(v_{1}, \ldots, v_{p}\right)$, which we triangulate by coning off to $v_{1}$ the triangulation on $\Delta \cap P$ coming from the constructed triangulation of $\Delta$, together with the triangulation of $P \cap\left[v_{0}, v_{2}, \ldots, v_{p}\right]$ obtained by induction on $p$, applying the present construction to the $(p-2)$-simplex $\Sigma_{2} \subset \cdots \subset \Sigma_{p}$.

Now we have a triangulation $T$ of $\Delta^{p}$ with the property that the vertices of each simplex correspond to compatible sphere systems, either $S_{j_{1}, \ldots, j_{p}}$ 's or $\Sigma_{i}$ 's. The barycentric subdivision of $T$ therefore maps to $\widehat{X}$. The face $\left[v_{1}, \ldots, v_{p}\right]$ corresponds to $\Sigma_{1} \subset \cdots \subset \Sigma_{p}$ which is already a simplex of $X$, so barycentrically subdividing $\left[v_{1}, \ldots, v_{p}\right]$ is not necessary. Thus we subdivide $T$ by starring at barycenters of all simplices not in $\left[v_{1}, \ldots, v_{p}\right]$, and we still have a simplicial map to $\widehat{X}$. Composing with the projection $\widehat{X} \rightarrow X$ gives the desired map $f$.

Remark 4.1. It is clear that the maps $f$ constructed in this way for varying $\Sigma_{1} \subset \cdots \subset \Sigma_{p}$ and fixed $S$ fit together on common faces to give a retraction of the cone on $X$ to $X$, hence a contraction of $X$ to the vertex $S$. 
Contractibility of $X$ was first proved in [C-V] in the case of no puncture. In either case, it is an easy consequence of the fact proved in $[\mathbf{H}]$ that the complex of all sphere systems is contractible.

In order to estimate the isoperimetric function for $X$, we now count the number of simplices in $T$.

Lemma 4.2. $T$ has $k_{1}^{p}+k_{2}^{p-1}+\cdots+k_{p}+1 p$-simplices.

Proof. We count using induction on $p$. The simplex $\Delta$ is contained in a cube of side length $k_{1}$. Each unit cube in this larger cube is subdivided into $p$ ! $p$-simplices, so the large cube contains $k_{i}^{p} p ! p$-simplices. It is also subdivided into $p$ ! large $p$-simplices like $\Delta$, so $\Delta$ contains $k_{1}^{p} p$-simplices of $T$. The prism $P\left(v_{1}, \ldots, v_{p}\right)$ was triangulated by coning off to the vertex $v_{1}$, so the number of $p$-simplices in it equals the number of $(p-1)$-simplices in the link of $v_{1}$, which equals the number of $(p-1)$-simplices in the restriction of $T$ to the face $\left[v_{0}, v_{2}, \ldots, v_{p}\right]$. By induction, this number is $k_{2}^{p-1}+\cdots+k_{p}+1$, so that $T$ has $k_{1}^{p}+k_{2}^{p-1}+\cdots+k_{p}+1 p$-simplices.

Theorem 4.3. The p-dimensional isoperimetric function for the complex $X$ is at most exponential.

Proof. Let $g: S^{p-1} \rightarrow X$ be simplicial with respect to a triangulation of $S^{p-1}$ into $N(p-1)$-simplices, and fix a vertex $S$ of the image of $g$. The contraction of $X$ to $S$ restricts to a contraction of the image of $g$ to $S$. Every vertex of $S^{p-1}$ can be joined to the base vertex by an edge-path in $S^{p-1}$ of length less than $N$, so by Proposition 3.4, the numbers $k_{i}$ for the various simplices in the image of $g$ are bounded by a number of the form $a^{N}$, where $a$ is a constant which depends only on $n$. By Lemma 4.2, the number of $p$-simplices necessary to cone off a single simplex is at most $a^{p N}$, so that the contraction of $g$ takes place in a subcomplex of $X$ containing at most $N a^{p N}$ $p$-simplices.

Corollary 4.4. The p-dimensional isoperimetric functions for the groups $\operatorname{Aut}\left(F_{n}\right)$ and $\operatorname{Out}\left(F_{n}\right)$ are at most exponential, for $n \geq 3$.

Proof. The $p$-dimensional isoperimetric function for a group $\Gamma$ may be computed by computing the corresponding function for any $(p-1)$-connected CW-complex quasi-isometric to $\Gamma$. ([A], [Gr2]). Thus it suffices to check that $X$ is contractible, locally finite and the action of $\Gamma$ on $X$ has finite simplex-stabilizers and finite quotient $X / \Gamma$ (see [Gh], Prop. 1.1).

We have already seen (see Remark 4.1) that $X$ is contractible. Local finiteness of $X$ follows from the fact that the complementary regions of a simple sphere system $S$ are punctured balls, and in a punctured ball there 
are only finitely many isotopy classes of embedded 2-spheres, determined by how they separate the punctures.

If $\Gamma_{\sigma}$ is the stabilizer of a simplex $\sigma=S_{0} \subset S_{1} \subset \ldots \subset S_{k}$, then $\Gamma_{\sigma}$ is contained in the stabilizer of the system $S_{k}$. By passing to a finite-index subgroup of $\Gamma_{S_{k}}$ we may assume each component of $M-S_{k}$ is taken to itself by a diffeomorphism which fixes each of its boundary spheres. Such diffeomorphisms are isotopic to the identity modulo twists along the spheres of $S_{k}$, since the complementary regions are punctured 3-balls. This shows that the stabilizer $\Gamma_{S_{k}}$, and hence $\Gamma_{\sigma}$, is finite.

Minimal simple sphere systems $S$ have $M-S$ connected and simplyconnected, and it is a standard 3-manifold fact that any two such systems are equivalent under a diffeomorphism of $M$. Since $M-S$ is a punctured ball, a minimal simple system is contained in only finitely many larger systems, and finiteness of $X / \Gamma$ follows.

\section{Mapping class groups.}

Given any punctured orientable surface with fundamental group $F_{n}$, the mapping class group $\Gamma$ of the surface is the subgroup of $\operatorname{Out}\left(F_{n}\right)$ which stabilizes the set of cyclic words in $F_{n}$ represented by simple loops around the punctures. There is a natural subcomplex of the sphere complex $X$ for $\operatorname{Out}\left(F_{n}\right)$ which is stabilized by $\Gamma$, and which can be described in terms of the surface as follows. Enlarge each of the punctures to a circle to form a compact surface $N$ with boundary. By an arc in $N$ we mean an imbedded arc with endpoints in the boundary of $N$. An arc system is a set of disjoint arcs, with no two parallel, i.e. isotopic by an isotopy of the pair $(N, \partial N)$, and none trivial, i.e. isotopic to an arc in the boundary. Isotopy classes of arc systems form a simplicial complex $\mathcal{A}(N)$, where a $k$-simplex is an arc system with $k+1$ arcs; again, isotopy is required to preserve, but not fix, the boundary.

An arc system $A$ is simple if each component of $N-A$ is a disk. The complex $X(N)$ is defined to be the geometric realization of the partially ordered set of isotopy classes of simple arc systems, so is a subcomplex of the barycentric subdivision of $\mathcal{A}(N)$. Contractibility of both $\mathcal{A}(N)$ and $X(N)$ follows from work of Harer [Ha1, Ha2]. See [H2] for a proof in the spirit of the present paper that $\mathcal{A}(N)$ is contractible; this proof can easily be modified to show that the subcomplex $X(N)$ is also contractible.

Alternatively, we now describe a natural embedding of $X(N)$ in a sphere complex $X=X(M)$ for a suitable $M$, such that the contraction of $X$ constructed in the previous section restricts to a contraction of $X(N)$. If we "fatten up" $N$ to a handlebody $N \times I$, then double it by gluing two copies 
together by the identity on the boundary $\partial(N \times I)$, we obtain a closed 3manifold $M$ which is a connected sum of $S^{1} \times S^{2}$ 's. Associating to each arc $\alpha$ in $N$ the 2-sphere $(\alpha \times I) \cup_{S^{1}}(\alpha \times I)$ in $M$ defines a simplicial map from $\mathcal{A}(N)$ to the sphere complex $\mathcal{S}(M)$ which takes $X(N)$ to $X=X(M)$. This "spherification" map $\sigma: X(N) \rightarrow X$ is injective since the injective map assigning to each arc complex the dual simplicial $\mathbf{R}$-tree factors through $\mathcal{S}(M)$.

The contractions of $X$ and $X(N)$ depend on normal form, which for arc systems is a classical notion considerably less subtle than for sphere systems: an arc system $A$ is in normal form with respect to an arc system $B$ if it has first been isotoped to minimize the number of points of $A \cap B$, and then $\operatorname{arcs}$ of $A$ isotopic to arcs of $B$ have been isotoped to coincide with these arcs. Such a normal form is unique up to isotopy through normal form systems, in contrast with the weaker notion of equivalence for normal forms of sphere systems. It is not hard to check that the spherification map $\sigma: X(N) \rightarrow X$ takes normal form arc systems to normal form sphere systems.

We claim that the combing/contraction of $X$ constructed in the previous section restricts to a combing/contraction of the subcomplex $X(N)$, i.e. the cones constructed in $\widehat{X}$ on simplices of $\widehat{X}(N)$ are entirely contained in $\widehat{X}(N)$. To see this, let $A$ and $B$ be arc systems and consider the innermost-circle surgery process applied to the sphere systems $S=\sigma(A)$ and $\Sigma=\sigma(B)$. The surgery has the same effect as pushing arcs of $A$ "across the ends" of $B$, then applying $\sigma$ to the resulting arc system. This process of surgering arcs is very much like the one in [H2], the only difference being that in [H2] one pushes arcs of $A$ across only one end of each arc of $B$, whereas the surgery process for spheres dictates pruning all ends of the trees of $T(\sigma(A))$ simultaneously. Note that for spherifications of arc systems these trees are homeomorphic to intervals, with no branching.

The mapping class group $\Gamma$ acts naturally on the contractible complex $X(N)$ with finite stabilizers and finite quotient, so $X(N)$ is quasi-isometric to $\Gamma$. The proof in the previous section that $\operatorname{Out}\left(F_{n}\right)$ satisfies exponential isoperimetric inequalities in all dimensions therefore restricts to a proof for $\Gamma$.

However, for $\Gamma$ one can get more information by combing in the opposite direction, from an arbitrary simple arc system $A$ towards the fixed simple arc system $B$ as follows. We first fix an ordering $\beta_{1}, \beta_{2}, \ldots$ on the $\operatorname{arcs}$ of $B$ and choose an endpoint $x_{i}$ for each $\beta_{i}$. Assuming $A$ is in normal form with respect to $B$, let $i$ be the smallest index such that $A$ meets $\beta_{i}$ transversely in at least one point. Then surger $A$ by sliding the point of $A \cap \beta_{i}$ closest to $x_{i}$ along $\beta_{i}$ to $x_{i}$, then separating the $\operatorname{arc}$ of $A$ which now meets $x_{i}$ into two disjoint arcs which do not intersect $\beta_{i}$ near $x_{i}$. In terms of spherifications this 
is surgering $\sigma(A)$, using the innermost circle of $\sigma(A) \cap \sigma(B)$ which bounds a disk containing $\sigma\left(x_{i}\right)$. Any trivial or duplicate arcs produced by the surgery are discarded. The resulting arc system can be perturbed to be disjoint from $A$, so $A$ is joined to this new system by a path of length at most two in $X(N)$. This is the first segment of the combing of $A$ toward $B$, and the process is then repeated until an arc system is produced which is disjoint from $B$ except for arcs they have in common. This system is then joined to $B$ by a path of length at most two in $X(N)$, completing the combing path for $A$. This combing is a combinatorial analog of the contraction process of [H2].

Proposition 5.1. The combing of $X(N)$ described above is asynchronously bounded, and induces an asynchronous bounded combing of $\Gamma$.

Proof. Fix the simple arc system $B$, and let $A \subset A^{\prime}$ be two simple arc systems which form an edge in $X(N)$. We claim that the combing paths from $A$ to $B$ and from $A^{\prime}$ to $B$ stay within distance two of each other in $X(N)$.

Ordering and orienting the arcs of $B$ has the effect of ordering the points of transverse intersection of $A^{\prime}$ with $B$ and the subset of transverse intersection points of $A$ and $B$. The surgeries which produce the combing paths eliminate these intersection points in order.

Several surgeries on arcs of $A^{\prime}$ may be required before we get to the first point in $A \cap B$. Each of these surgeries produces an arc system which contains $A$. The next surgery, eliminating a point of $A \cap B$, produces an arc system $A_{1}^{\prime}$ which is compatible with $A$ and contains the system $A_{1}$ obtained by surgering $A$ to eliminate this point. The surgery process then proceeds from $A_{1} \subset A_{1}^{\prime}$.

The edge paths of $\mathcal{A}(N)$ produced by these surgeries are related by a simplicial homotopy as shown in Figure 3 . The edge path for $A^{\prime}$ runs horizontally across the top, while that for $A$ runs across the bottom. After barycentric subdivision we have a simplicial homotopy in $X(N)$ between the combing paths for $A$ and $A^{\prime}$, in which the paths remain at most two edges apart.

The complex $X(N)$ is quasi-isometric to $\Gamma$, and existence of an asynchronous bounded combing is a quasi-isometry invariant.

The difficulty with extending this proof to $\operatorname{Aut}\left(F_{n}\right)$ is that the component trees of a graph $T(S)$ can have branching, so one cannot always prune them from a fixed endpoint. One is more or less forced to prune all free ends simultaneously, if the process is to be at all canonical. But for a pair $S \subset S^{\prime}$, the trees of $T(S)$ need not look much like the trees of $T\left(S^{\prime}\right)$, so the sequence of canonical prunings for $T(S)$ may not be very closely related to the sequence 
for $T\left(S^{\prime}\right)$.

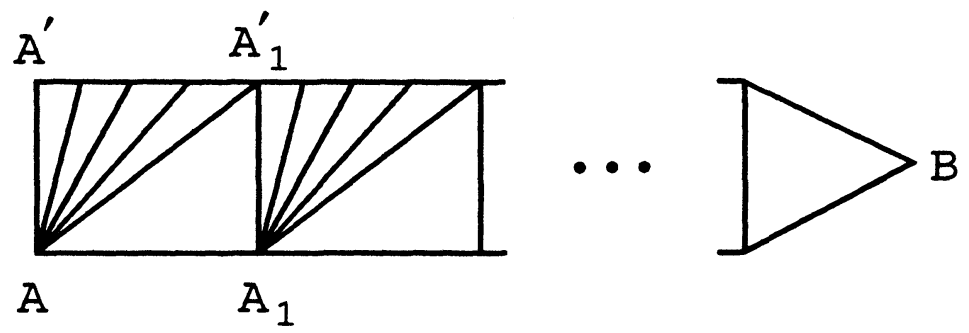

\section{Figure 3.}

This proposition gives an alternative proof that $\Gamma$ satisfies an exponential isoperimetric inequality, since this is true for any group with an asynchronous bounded combing ([ECHLPT], p. 154).

\section{References}

[A] J. Alonso, Inegalités isopérimetriques et quasi-isometries, C.R. Acad. Sci. Paris, 311(I) (1990), 761-764.

[AB] J. Alonso and M. Bridson, Semihyperbolic groups, Proc. London Math. Soc., 70(1) (1995), 56-114.

[BV] M. Bridson and K. Vogtmann, On the geometry of the group of automorphism of a free group, Bull. London Math. Soc., 27 (1995), 544-552.

[CV] M. Culler and K. Vogtmann, Moduli of graphs and automorphisms of free groups, Invent. Math, 84 (1986), 91-119.

[ECHLPT] D. Epstein, J. Cannon, D. Holt, S. Levy, M. Paterson and W. Thurston, Word Processing in Groups, London, Jones and Bartlett, (1992).

[Ge] S.M. Gersten, The automorphism group of a free group is not a CAT(0) group, Proc. A.M.S., 121(4) (1994), 999-1002.

[Ge2] - Isoperimetric and isodiametric functions of finite presentations, in Geometric Group Theory, ed. by G. Niblo and M. Roller, London Math Soc. Lecture Notes, Cambridge University Press, 181(2) (1993), 79-96.

[Gh] E. Ghys, Les groupes hyperboliques, Seminaire Bourbaki, 722 (1989-90).

[Gr] M. Gromov, Hyperbolic groups, in Essays in Group Theory, ed. by S. M. Gersten, M.S.R.I., Springer-Verlag, 8 (1987), 75-263.

[Gr2] , Asymptotic invariants of groups, in Geometric Group Theory, ed. by G. Niblo and M. Roller, London Math Soc. Lecture Notes, Cambridge University Press, 181(2) (1993).

[Ha1] J. Harer, Stability for the homology of mapping class groups of orientable surfaces, Annals of Math., 121(2) (1985), 2215-249.

[Ha2] - The virtual cohomological dimension of the mapping class group of an oriented surface, Invent. Math., 84 (1986), 157-176.

[H] A. Hatcher, Homological stability for automorphism groups of free groups, Comment. Math. Helv., 70 (1995), 39-62. 
[H2] — On triangulations of surfaces, Top. and its Appl., 40 (1991), 189-194.

[L] F. Laudenbach, Topologie de la dimension trois: homotopie et isotopie, Astérisque 12, Soc. Math. France (1974).

[L2] - Sur les 2-spheres d'une variété de dimension 3, Annals of Math., 97 (1973), $57-81$.

[M1] L. Mosher, Mapping class groups are automatic, Math. Res. Letters, 1(2) (1994) 249-256.

[M2] — Mapping class groups are automatic, Annals of Math., 142 (1995), 303-384.

[S] J.-P. Serre, Arbres, amalgames, $S L_{2}$, Astérisque, 46, Soc. Math. France (1977).

Received October 25, 1993 and revised May 5, 1995. The authors were partially supported by NSF grant DMS-9307313.

CORNELl UNIVERSITY

ITHACA, NY 14853-7901

E-mail address: vogtmann@math.cornell.edu 

Peng Lin and Richard Rochberg, Trace ideal criteria for Toeplitz and Hankel operators on the weighted Bergman spaces with exponential type

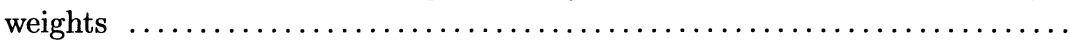

Donald E. Marshall and Arne Stray, Interpolating Blaschke products . .

Kathy D. Merrill and Lynne $\mathbf{H}$. Walling, On quadratic reciprocity over

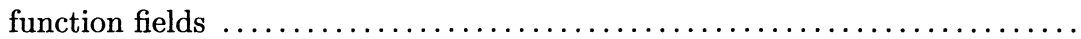

Takahiko Nakazi and Masahiro Yamada, $\left(A_{2}\right)$-conditions and Carleson

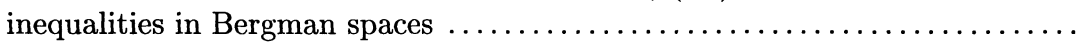

C. Ott, A note on a paper of E. Boasso and A. Larotonda ..............

Victor Patrangenaru, Classifying 3 and 4 dimensional homogeneous Riemannian manifolds by Cartan triples

Carlo Pensavalle and Tim Steger, Tensor products with anisotropic principal series representations of free groups

Ying Shen, On Ricci deformation of a Riemannian metric on manifold with boundary

Albert Jeu-Liang Sheu, The Weyl quantization of Poisson $S U(2) \ldots \ldots$

Alexandra Shlapentokh, Polynomials with a given discriminant over fields of algebraic functions of positive characteristic

Eric Stade and D.I. Wallace, Weyl's law for $S L(3, \mathbb{Z}) \backslash S L(3, \mathbb{R}) / S O(3, \mathbb{R})$

Christopher W. Stark, Resolutions modeled on ternary trees ........ 557

Per Tomter, Minimal hyperspheres in two-point homogeneous spaces .....

Jun Tomiyama, Topological Full groups and structure of normalizers in

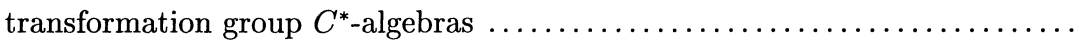

Nik Weaver, Subalgebras of little Lipschitz algebras 


\section{PACIFIC JOURNAL OF MATHEMATICS}

\section{Volume $173 \quad$ No. $2 \quad$ April 1996}

A mean value inequality with applications to Bergman space operators

PATRICK ROBERT AHERn and ZELJKO CUCKOVIC

$H^{p}$-estimates of holomorphic division formulas

MATS ANDERSSON and HASSE CARLSSON

Group structure and maximal division for cubic recursions with a double root

Christian JeAn-Claude Ballot

The Weil representation and Gauss sums

ANTONiA WiLson BLUHER

Duality for the quantum $E(2)$ group

ALFONS VAN DAELE and S. L. WORONOWICZ

Cohomology complex projective space with degree one codimension-two fixed submanifolds 387

KARL HEINZ DOVERMANN and ROBERT D. LITTLE

On the mapping intersection problem

ALEXANDER DRANISHNIKOV

From the $L^{1}$ norms of the complex heat kernels to a Hörmander multiplier theorem for

sub-Laplacians on nilpotent Lie groups

\section{XUAN THINH DUONG}

Isoperimetric inequalities for automorphism groups of free groups

Allen E. Hatcher and Karen Vogtmann

Approximation by normal elements with finite spectra in $C^{*}$-algebras of real rank zero

HUAXIN LIN

Interpolating Blaschke products

DonALD EdDY MARShall and ARNE STRAY

Interpolating Blaschke products generate $H^{\infty}$

JOHN BRADY GARNETT and ARTUR NICOLAU

Classifying 3- and 4-dimensional homogeneous Riemannian manifolds by Cartan triples

VICTOR PATRANGENARU

Polynomials with a given discriminant over fields of algebraic functions of positive

characteristic

ALEXANDRA SHLAPENTOKH

Resolutions modeled on ternary trees

CHRISTOPHER W. STARK

Topological full groups and structure of normalizers in transformation group $C^{*}$-algebras 